\title{
Condición física y bienestar emocional en escolares de 7 a 12 años
}

\author{
Andrés Rosa Guillamón, Eliseo García Cantó* y Juan José Pérez Soto \\ Universidad de Murcia, España.
}

Recibido, septiembre 17/2017

Concepto de evaluación, diciembre 3/2017

Aceptado, marzo 8/2018

Referencia: Rosa Guillamón, A., García Cantó, E. \& Pérez Soto, J.J. (2018). Condición física y bienestar emocional en escolares de 7 a 12 años. Acta colombiana de Psicología, 21(2), 282-291. doi: http://www.dx.doi. org/10.14718/ACP.2018.21.2.13

\section{Resumen}

\begin{abstract}
El incremento de la prevalencia de conductas y trastornos que pueden afectar gravemente el bienestar emocional infantil es un hecho. Sin embargo, aunque la relación causal entre condición física y salud biológica se encuentra bien establecida, la asociación entre la condición física y algunas dimensiones de la salud psicológica es menos evidente. El objetivo de esta investigación fue analizar la relación entre la condición física y el bienestar emocional de escolares. Se realizó un estudio inferencial mediante análisis de la covarianza ajustada por edad en una muestra de 212 escolares (120 mujeres) de 7 a 12 años de edad, seleccionada por conveniencia, en la que se aplicó la Batería ALPHA-fitness para medir la condición física y el Strengths and Difficulties Questionnaire para evaluar el bienestar emocional. Los resultados mostraron que los participantes con mayor condición física $(\mathrm{X} \geq \mathrm{P} 50)$ obtuvieron valores más positivos en problemas de conducta $(\mathrm{p}=.002)$, hiperactividad $(\mathrm{p}<.001)$, problemas con compañeros $(\mathrm{p}=.028)$, conducta prosocial $(\mathrm{p}<.001)$, y puntuación total de dificultades $(\mathrm{p}=.029)$; mientras que los escolares con menor nivel en la puntuación total de dificultades $(\mathrm{X}<\mathrm{P} 40)$ tuvieron un mejor rendimiento en $4 \times 10 \mathrm{~m}(\mathrm{p}=.010)$, dinamometría manual $(\mathrm{p}=.050)$, Course-Navette $(\mathrm{p}=.050)$ y condición física general $(\mathrm{p}=.040)$. Estos resultados sugieren una relación positiva y bidireccional entre la condición física y el bienestar emocional; y, por tanto, se refuerza la necesidad de impulsar programas de mejora de la salud que tengan en cuenta la condición física y evalúen su efecto sobre las capacidades psicológicas de los escolares.

Palabras clave: condición física, actividad física, bienestar emocional, educación física, niños.
\end{abstract}

\section{Physical fitness and emotional well-being in school children aged 7 to 12 years}

\begin{abstract}
The increase in the prevalence of behaviors and disorders severely affecting children's emotional well-being is a fact. The causal relationship between fitness and biological health is well established. However, the relationship between fitness and some dimensions of psychological health is less evident. The aim of this paper was to analyze the relationship between fitness and emotional well-being. The ALPHA-fitness Battery was used to assess fitness, and the Strengths and Difficulties Questionnaire, to assess emotional well-being. A convenience sample of 212 school children (120 women) aged 7-12 years was used. An inferential study was carried out by means of covariance analysis adjusted by age. The results showed that those children with higher fitness $\left(\mathrm{X} \geq \mathrm{P}_{50}\right)$ had more positive values in behavior problems $(p=0,002)$, hyperactivity $(p<0,001)$, problems with partners $(p=0,028)$, prosocial behavior $(p<0,001)$ and total score of difficulties $(p=0,029)$. Students with a lower level of total difficulties score $\left(\mathrm{X}<\mathrm{P}_{40}\right)$ had a better performance in $4 \times 10 \mathrm{~m}(p=0,010)$, manual dynamometry $(p=0,050)$, Course-Navette $(p=0,050)$ and general fitness $(p=0,040)$ with respect to those with medium and high levels. These results suggest a positive and bidirectional relationship between fitness and emotional well-being in primary school children. Therefore, it reinforces the need to promote programs to improve health related fitness and assess their effect on the psychological abilities of school children.

Key words: fitness, physical activity, emotional well-being, physical education, children
\end{abstract}

\footnotetext{
Campus Universitario de Espinardo. Facultad de Educación. CP: 30100, Espinardo (Murcia, España). Tel.: +0034 868887088. eliseo.garcia@um.es
} 


\title{
Condiçäo fisica e bem-estar emocional em estudantes de 7 a 12 anos
}

\begin{abstract}
Resumo
O aumento da prevalência de consultas e transtornos que podem afetar gravemente o bem-estar emocional infantil é um fato. No entanto, ainda que a relação causal entre condição física e saúde biológica esteja bem estabelecida, a associação entre a condição física e algumas dimensões da saúde psicológica é menos evidente. O objetivo desta pesquisa foi analisar a relação entre a condição física e o bem-estar emocional de crianças em idade escolar. Realizou-se um estudo inferencial mediante a análise da covariância ajustada por idade em uma amostra de 212 estudantes (120 mulheres) de 7 a 12 anos de idade, selecionada por conveniência, na qual aplicou-se a bateria ALPHA-Fitness para medir a condição física e o Strengths and Difficulties Questionnaire para avaliar o bem-estar emocional. Os resultados mostraram que os participantes com maior condição física $(X \geq P 50)$ obtiveram valores mais positivos em problemas de conduta $(p=.002)$, hiperatividade $(p<.001)$, problemas com colegas $(\mathrm{p}=.028)$, conduta pró-social $(\mathrm{p}<.001)$ e pontuação total de dificuldades $(\mathrm{p}=.029)$; enquanto os estudantes com menor nível na pontuação total de dificuldades $(X<P 40)$ tiveram um melhor rendimento em 4 x $10 \mathrm{~m}$ $(\mathrm{p}=.010)$, dinamometria manual $(\mathrm{p}=.050)$, Course-Navette $(\mathrm{p}=.050)$ e condição física geral $(\mathrm{p}=.040)$. Esses resultados sugerem uma relação positiva e bidirecional entre a condição física e o bem-estar emocional e, portanto, reforça-se a necessidade de impulsionar programas de melhoria da saúde que levem em consideração a condição física e avaliem seu efeito sobre as capacidades psicológicas das crianças em idade escolar.

Palavras-chave: atividade física, bem-estar emocional, condição física, crianças, educação física.
\end{abstract}

\section{INTRODUCCIÓN}

La evidencia científica disponible indica que la realización de actividad física habitual y de manera moderada ( $\geq 3$ METS) a fuerte $(\geq 6$ METS) no solo favorece la salud psicobiológica de los individuos (Blair, 2009; Ortega, Ruiz \& Castillo, 2013; Rosa, García-Cantó, Rodríguez-García \& Pérez-Soto, 2017a), sino que constituye un factor de primer orden como tratamiento coadyuvante y preventivo de enfermedades en adultos mayores (Mavrovouniotis, Argiriadou \& Papaioannou, 2010), adultos (Hackney \& Earhart, 2010) y jóvenes (Jeong et al., 2005).

A nivel fisiológico, la actividad física representa una de las estrategias más económicas y eficaces en la lucha contra la obesidad infanto-juvenil (Gálvez et al., 2015a; Gálvez et al., 2015b; Gálvez et al., 2015c), y a nivel psicosocial puede contribuir a la disminución de los niveles percibidos de depresión, cansancio, ansiedad y estrés; y mejorar la atención, la autoestima, el estado de ánimo y el autoconcepto en adultos y jóvenes (Babiss \& Gangwisch, 2009; Bernstein \& Mc Nally, 2017; Jiménez, Martínez, Miró \& Sánchez, 2008; Panagiotopoulos et al., 2011).

A pesar de que existe cierta controversia entre la comunidad científica (Martínez-Vizcaíno \& Sánchez-López, 2008), la evidencia apunta a que la actividad física sistematizada puede incrementar los niveles de condición física (CF) en niños y jóvenes (Dencker et al., 2006), pudiendo ser esta una relación bidireccional (Rosa et al., 2017b).
Específicamente, la condición física se define como la capacidad para realizar actividad física (o ejercicio físico) (Ortega et al., 2013), y su valoración representa una medida que describe el estado integrado de las principales funciones orgánicas que intervienen en el movimiento corporal, como la musculoesquelética, la cardiorrespiratoria, la hematocirculatoria, la endocrinometabólica y la psiconeurológica (Gálvez et al., 2015b).

En la literatura científica, la relación causal entre la condición física y la salud biológica se encuentra bien establecida (Ortega et al., 2009; Ortega et al., 2013); no obstante, la relación entre la condición física y otras dimensiones de la salud psicológica-como las emociones, pensamientos y el estado de ánimo, entre otras- es menos evidente, e incluso se observan algunos resultados contradictorios (Berstein \& Mc Nally, 2017; Rosa-Guillamón \& García-Cantó, 2016; Rosa, García-Cantó, Rodríguez-García \& Pérez-Soto, 2016; Rosa et al., 2017a). Al respecto, cabe aclarar que la salud psicológica es entendida como el estado de bienestar en el cual el individuo es consciente de sus propias capacidades, además de que puede afrontar las tensiones normales de la vida, puede trabajar de forma productiva y fructífera, y es capaz de hacer una contribución a su comunidad (Organización Mundial de la Salud, 2013); y que una de las dimensiones de la salud psicológica es el bienestar emocional, definido por Diener, Suh, Lucas y Smith (1999) como una "construcción individual" que incluye medidas positivas -como el afecto positivo, la felicidad, la satisfacción vital, 
y otras- y medidas negativas - como el afecto negativo, la ansiedad, el estrés y la depresión, entre otras-.

Al respecto, en diversos estudios se ha descrito una relación directa entre la condición física y el bienestar emocional, principalmente en jóvenes (Jeoung, Hong \& Lee, 2013); así como una asociación entre la capacidad aeróbica y la felicidad subjetiva (Jiménez-Moral, Zagalaz, Molero, Pulido-Martos \& Ruiz, 2013), y entre la capacidad aeróbica y la satisfacción vital (Padilla-Moledo et al., 2012).

Asimismo, en escolares de primaria se ha encontrado una relación entre la condición física y algunos constructos que influyen en el bienestar emocional, como lo son la calidad de vida percibida (Gálvez et al., 2015a; Rosa-Guillamón et al., 2016), el autoconcepto general (Gálvez et al., 2016; Rodríguez-García et al., 2014), el autoconcepto social y conductual (Rodríguez-García et al., 2015), y la ansiedad social (Gálvez et al., 2016).

Sin embargo, aunque la sabiduría coloquial y algunos estudios sugieren una fuerte asociación entre la actividad física regular, la condición física y el bienestar emocional (Biddle \& Asare, 2011; Philipsson, Duberg, Moller \& Hagberg, 2013; Soyeon \& Fedewa, 2011; West, Otte, Geher, Johnson \& Mohr, 2004), la naturaleza de este vínculo continúa siendo poco comprendida (Berstein \& Mc Nally, 2017), especialmente en la infancia (Rosa-Guillamón \& García-Cantó, 2016; Rosa et al., 2016).

A esto se le añade que el incremento de la prevalencia de desórdenes psicosociales que pueden afectar gravemente el bienestar emocional infantil es un hecho que preocupa a investigadores e instituciones sanitarias y educativas (Navarro-Pardo, Meléndez, Sales \& Sancerni, 2012), debido a que los sujetos en edad escolar se encuentran en un complejo proceso de cambios a nivel fisiológico, psicológico y socioafectivo que pueden influir sobre la construcción de una personalidad equilibrada, así como en la adopción de conductas de ajuste social y en un estilo de vida autónomo y saludable en edades posteriores (Gálvez et al., 2015b; Rosa et al., 2016). Por tanto, intervenir mediante el incremento de las oportunidades de práctica física para mejorar su estado de condición física y bienestar emocional podría favorecer la adquisición de otros hábitos saludables (Rosa et al., 2017a, b).
A partir de lo anterior, en el presente artículo se plantea como hipótesis de estudio que los escolares con mejor condición física presentan una percepción más positiva de su bienestar emocional. Y, por tanto, el objetivo fue analizar la relación entre la condición física y el bienestar emocional en una muestra de escolares de enseñanza primaria.

\section{MÉTODO}

\section{Diseño}

Se llevó a cabo un estudio empírico observacional, de corte transversal y ex post facto (Thomas \& Nelson, 2007).

\section{Participantes}

Hicieron parte del estudio 212 escolares ( 92 hombres, 120 mujeres) de 7 a 12 años $(\mathrm{M}=9.59 ; \mathrm{DE}=1.09)$ de la Región de Murcia, España (véase Tabla 1) que pertenecían a cuatro colegios públicos (muestra por conveniencia). Acá, es importante mencionar que se solicitó por escrito el consentimiento informado por parte de los padres o tutores legales de cada uno de los niños, y que como criterio de exclusión se planteó la presencia de enfermedades mentales u óseas-articulares.

\section{Consideraciones éticas}

Esta investigación se desarrolló siguiendo las normas deontológicas reconocidas por la Declaración de Helsinki (revisión de 2013), y contó con la aprobación del comité de bioética de la Universidad de Murcia.

\section{Variables e instrumentos}

La condición física se midió por medio de la Batería ALPHA-Fitness basada en la evidencia de Ruiz et al. (2011), en la que se incluyeron los indicadores de (a) fuerza del tren superior -dinamometría manual-, (b) fuerza del tren inferior -salto longitudinal-, y (c) capacidad aeróbica-Course-Navette-, exceptuando la medición de grasa corporal -por motivos de disponibilidad de tiempo-; y a esto se le añadió el test de 4 x 10 m-velocidad-agilidad-.

Tabla 1

Distribución de la muestra

\begin{tabular}{lllllll}
\hline & 7 años & 8 años & 9 años & 10 años & 11 años & 12 años \\
\hline Varones, $\mathrm{n}(\%)$ & $21(22.8)$ & $10(1.9)$ & $4(4.3)$ & $9(9.8)$ & $39(42.4)$ & $9(9.8)$ \\
Mujeres, $\mathrm{n}(\%)$ & $17(14.2)$ & $12(1.0)$ & $15(12.5)$ & $15(12.5)$ & $53(44.2)$ & $8(6.7)$ \\
\hline
\end{tabular}


Las pruebas seleccionadas permiten una valoración objetiva y completa de la condición física (Ortega et al., 2005), tienen una relación directa con la salud -según los postulados del American College of Sport Medicine (Amstrong, Whaley, Brubaker \& Otto, 2005)-, han sido descritas en otros trabajos (Gálvez et al., 2015b; Rosa et al., 2016; Rosa et al., 2017), y cuentan con pruebas de fiabilidad y validez en poblaciones con características sociodemográficas similares a las de esta investigación, donde se encuentran valores en el coeficiente de correlación intraclase e interexplorador entre .95 y .99 (Rosa, Rodríguez-García, García-Cantó \& Pérez-Soto, 2015).

Adicionalmente, se creó la variable CF-ALPHA, que representa la suma de la media escalada de las puntuaciones en cada prueba de condición física (Rodríguez-García et al., 2014; Rosa et al., 2016), a partir de la cual se categorizó a los participantes en dos niveles de condición física, ajustados en función del sexo: $\mathrm{X}<\mathrm{P} 50=$ menor condición física $(\mathrm{mCF}), \mathrm{y} X \geq \mathrm{P} 50=$ mayor condición física $(\mathrm{MCF})$ (Rosa-Guillamón \& García-Cantó, 2016). Específicamente, los hombres obtuvieron un P50 de 5.75, mientras que en las mujeres fue de 4.50 .

Por otra parte, el bienestar emocional se evaluó con el cuestionario Strengths and Difficulties Questionnaire (SDQ) de Goodman (1997), que suele emplearse para evaluar diferentes problemas emocionales y comportamentales relacionados con las capacidades psicológicas en sujetos de 4 a 16 años de edad, tomando como criterio de referencia los últimos seis meses. Específicamente, este instrumento está compuesto por 25 ítems distribuidos en cinco subescalas: síntomas emocionales, problemas conductuales, problemas con compañeros, hiperactividad y conducta pro-social; y cada ítem presenta tres modalidades de respuesta ("no es verdad", "es verdad a medias" y "verdaderamente sí,, que se puntúan con cero, uno y dos, respectivamente). La escala de aspectos positivos (conducta prosocial) se puntúa a la inversa. Cada escala se puntúa entre cero y 10.

La suma de los registros de las cinco escalas da como resultado una valoración global denominada "puntuación total de dificultades" (PTD), con valores entre cero y 40, en donde un puntaje elevado es indicativo de una mayor sintomatología afectiva y comportamental. En este estudio, los escolares fueron categorizados en tres grupos según su nivel de PTD: nivel bajo $=\mathrm{X}<\mathrm{P}_{40}$; nivel medio $=\mathrm{P}_{40} \leq \mathrm{X}>\mathrm{P}_{80}$; $\mathrm{y}$ nivel alto $=\mathrm{X} \geq \mathrm{P}_{80}$.

La validez y fiabilidad del SDQ ha sido demostrada en diversos estudios (Goodman, Ford, Simmons, Gatward \& Meltzer, 2000a; Goodman, Renfrew \& Mullick, 2000b; Goodman \& Scott, 1999; Ruchkin, Jones, Vermeiren \& Schwab-Stone, 2008), y en este trabajo se aplicaron pruebas de fiabilidad para los 25 ítems de la escala, así como un análisis factorial confirmatorio para verificar la agrupación de los ítems en las distintas subescalas definidas originalmente (Goodman, 1997). Con esto, se encontró que todos los ítems de la prueba poseen consistencia y fiabilidad, ya que de ser eliminado alguno de ellos, disminuye la varianza explicada y la fiabilidad global en cada subconstructo y en la escala global.

Específicamente, la escala global de PTD arrojó una buena fiabilidad ( $\alpha=.892$ ), y la fiabilidad de los diferentes subconstructos fue de .876 para síntomas emocionales, .856 para problemas conductuales, .923 para problemas con los compañeros, .798 para hiperactividad y .865 para conducta prosocial; mientras que la varianza explicada por cada uno de los subconstructos fue de $12.45 \%$ para síntomas emocionales, $15.45 \%$ para problemas conductuales, $23.64 \%$ para problemas con los compañeros, $11.34 \%$ para hiperactividad y $14.67 \%$ para conducta prosocial. La varianza total explicada fue de $77.88 \%$.

\section{Procedimiento}

El trabajo de campo fue realizado durante el curso académico 2016/17, en horario lectivo, mediante visitas a los centros durante el mes de febrero. El equipo de investigadores, formado por dos maestros de educación física y el investigador principal, realizó una sesión de entrenamiento teórico-práctica, para la cual se recomendó no realizar actividades deportivas la tarde anterior, no alterar la alimentación habitual y vestir ropa deportiva ligera, y posteriormente se realizó un calentamiento basado en movilidad articular dinámica. El SDQ fue administrado de manera colectiva en una sala que permitía privacidad y libertad en su cumplimentación. La duración media fue de 20 minutos.

\section{Análisis estadístico}

Inicialmente, se calcularon descriptivos básicos de las puntuaciones, y para constatar la distribución normal de los datos se aplicó la prueba de Kolmogorov-Smirnov. Posteriormente, las diferencias según sexo (hombres $=0 \mathrm{y}$ mujeres $=1$ ) se estudiaron mediante el análisis de la covarianza (ANCOVA) ajustado por edad (9.76 años) para las variables continuas, y el test de Chi-Cuadrado de Pearson para las variables categóricas. Asimismo, las diferencias en el bienestar emocional se estudiaron mediante un análisis de la covarianza (ANCOVA) ajustado por sexo, al igual que las diferencias en la condición física según la PTD. Finalmente, se estimó el tamaño del efecto por medio de la $\eta^{2}$, y la significancia estadística se fijó a un valor $p \leq .05$. Para el análisis, se utilizó el programa estadístico SPSS (v.19.0, Chicago, Illinois, EE. UU.). 


\section{RESULTADOS}

A continuación, se presentan los resultados del análisis descriptivo e inferencial de las variables de condición física y bienestar emocional. En la Tabla 2 se muestran las diferencias entre la condición física y el bienestar emocional según el sexo, y, como se puede observar, la prueba ANCOVA detectó diferencias estadísticamente significativas, ya que los hombres mostraron registros superiores en los test de $4 \times 10 \mathrm{~m}(p=.002)$, salto longitudinal $(p=.033)$, y Course-Navette, con respecto a las mujeres. De resto, no se encontraron diferencias significativas en las demás variables analizadas $(p>.1)$.

Por otra parte, en la Tabla 3 se presentan los resultados con respecto al bienestar emocional, según el nivel de condición física. Específicamente, la prueba ANCOVA reportó diferencias estadísticamente significativas, ya que los escolares con MCF-ALPHA mostraron registros superiores en las variables de síntomas emocionales $(p=.001)$, problemas con compañeros $(p=.015)$ y $\operatorname{PTD}(p=.013)$, con respecto a aquellos con mCF-ALPHA.

Finalmente, en la Tabla 4 se presentan los valores de los test de condición física, según el nivel de PTD. Acá, la prueba ANCOVA registró diferencias estadísticamente significativas, ya que aquellos que tenían un nivel alto de PTD presentaron menores registros en los test de $4 \times 10$ $\mathrm{m}(p=.010)$, dinamometría manual $(p=.050)$, CourseNavette $(p=.050)$ y CF-ALPHA $(p=.004)$, con respecto a sus semejantes con un nivel medio o bajo.

Tabla 2

Diferencias según sexo en las variables de estudio

\begin{tabular}{|c|c|c|c|c|c|}
\hline & $\begin{array}{l}\text { Hombres }(n=92) \\
M \pm D E(I C ~ 95 \%)\end{array}$ & $\begin{array}{l}\text { Mujeres }(n=120) \\
M \pm D E(I C 95 \%)\end{array}$ & $\mathrm{F}$ & valor $\mathrm{p}$ & $\eta 2$ \\
\hline $4 \times 10 \mathrm{~m}(\mathrm{~s})$ & $13.4 \pm 1.4(13.1-13.6)$ & $13.9 \pm 1.4(13.7-14.1)$ & -2.886 & .002 & .045 \\
\hline Dinamometría manual (kg) & $16.2 \pm 5.7(15.6-17.1)$ & $15.8 \pm 5.1(14.9-16.3)$ & -.370 & .149 & .010 \\
\hline Salto longitudinal (cm) & $105.2 \pm 2.2(101.3-109.5)$ & $99.7 \pm 21.0(95.9-103.1)$ & -2.237 & .033 & .021 \\
\hline Course-Navette (paliers) & $3.9 \pm 1.8(3.6-4.2)$ & $2.9 \pm 1.3(2.6-3.1)$ & -3.987 & $<.001$ & .101 \\
\hline CF-ALPHA & $5.9 \pm 2.1(5.7-6.4)$ & $4.9 \pm 2.1(4.6-5.2)$ & -3.368 & $<.001$ & .100 \\
\hline $\mathrm{mCF}, \mathrm{n}(\%)$ & $49(43.8)$ & $63(56.3)$ & $1.750 *$ & .120 & - \\
\hline MCF, n (\%) & $43(43.0)$ & $57(57.0)$ & $1.960 *$ & .162 & - \\
\hline Síntomas emocionales & $2.1 \pm 1.9(1.6-2.5)$ & $2.5 \pm 2.4(2.1-2.9)$ & 2.431 & .120 & .011 \\
\hline Problemas de conducta & $1.7 \pm 1.7(1.4-2.1)$ & $1.4 \pm 1.6(1.1-1.7)$ & 2.006 & .158 & .010 \\
\hline Hiperactividad & $3.2 \pm 2.1(2.8-3.7)$ & $3.1 \pm 2.2(2.7-3.5)$ & .210 & .648 & .001 \\
\hline Problemas con compañeros & $1.9 \pm 1.9(1.5-2.3)$ & $1.6 \pm 1.7(1.3-1.9)$ & 1.429 & .233 & .007 \\
\hline Conducta prosocial & $8.5 \pm 1.5(8.2-8.8)$ & $8.9 \pm 1.4(0.6-9.1)$ & 3.359 & .050 & .016 \\
\hline Puntuación Total de Dificultades & $9.0 \pm 5.2(7.8-10.1)$ & $8.6 \pm 5.5(7.7-9.6)$ & .140 & .709 & .001 \\
\hline Baja, n (\%) & $38(40.4)$ & $56(59.6)$ & $1.471^{*}$ & .225 & - \\
\hline Media, n (\%) & $35(46.1)$ & $41(53.9)$ & $.059 *$ & .808 & - \\
\hline Alta, n (\%) & $19(45.2)$ & $23(54.8)$ & $1.143 *$ & .285 & - \\
\hline
\end{tabular}

Nota. $(p<.001)$ y CF $(p<.001)$, conducta prosocial $(p=.050)$. CF-ALPHA = condición física; $\mathrm{mCF}=$ menor condición física; $\mathrm{MCF}=$ mayor condición física. ${ }^{*}$ Chi-cuadrado.

Tabla 3

Diferencias en el bienestar emocional según el nivel de condición física

\begin{tabular}{|c|c|c|c|c|c|}
\hline & $\begin{array}{c}\text { mCF-ALPHA }(\mathrm{n}=112) \\
\mathrm{M} \pm \mathrm{DE}(\mathrm{IC} 95 \%)\end{array}$ & $\begin{array}{c}\text { MCF-ALPHA }(\mathrm{n}=110) \\
\mathrm{M} \pm \mathrm{DE}(\mathrm{IC} 95 \%)\end{array}$ & $\mathrm{F}$ & valor $\mathrm{p}$ & $\eta 2$ \\
\hline Síntomas emocionales & $2.8 \pm 2.4(2.4-3.2)$ & $1.7 \pm 1.7(1.3-2.2)$ & 11.718 & .001 & .053 \\
\hline Problemas de conducta & $1.6 \pm 1.8(1.3-1.9)$ & $1.4 \pm 1.4(1.1-1.7)$ & .523 & .470 & .002 \\
\hline Hiperactividad & $3.2 \pm 2.2(2.8-3.6)$ & $3.1 \pm 2.1(2.7-3.6)$ & .024 & .877 & $<.001$ \\
\hline Problemas con compañeros & $2.1 \pm 1.7(1.7-2.4)$ & $1.4 \pm 1.8(1.1-1.8)$ & 5.970 & .015 & .028 \\
\hline Conducta prosocial & $8.7 \pm 1.4(8.4-9.1)$ & $8.7 \pm 1.5(8.4-9.1)$ & .022 & .883 & $<.001$ \\
\hline Puntuación total de dificultades & $9.6 \pm 5.7(8.6-10.6)$ & $7.8 \pm 4.9(6.7-8.8)$ & 6.280 & .013 & .029 \\
\hline
\end{tabular}

Nota. CF-ALPHA = condición física.; $\mathrm{mCF}=$ menor condición física; $\mathrm{MCF}=$ mayor condición física. 
Tabla 4

Diferencias en la condición física según el nivel de puntuación total de dificultades

\begin{tabular}{|c|c|c|c|c|c|c|}
\hline & $\begin{array}{c}\text { Bajo }(\mathrm{n}=94) \\
\mathrm{M} \pm \mathrm{DE}(\mathrm{IC} 95 \%)\end{array}$ & $\begin{array}{c}\text { Medio }(\mathrm{n}=76) \\
\mathrm{M} \pm \mathrm{DE}(\mathrm{IC} 95 \%)\end{array}$ & $\begin{array}{c}\text { Alto }(\mathrm{n}=92) \\
\mathrm{M} \pm \mathrm{DE}(\mathrm{IC} 95 \%)\end{array}$ & $\mathrm{F}$ & valor $\mathrm{p}$ & $\eta 2$ \\
\hline $4 \times 10 \mathrm{~m}(\mathrm{~s})$ & $13.5 \pm 1.3(13.2-13.7)$ & $13.6 \pm 1.4(13.3-13.9)$ & $14.3 \pm 1.4(13.8-14.7)$ & 4.678 & .010 & .043 \\
\hline $\begin{array}{l}\text { Dinamometría } \\
\text { manual }(\mathrm{kg})\end{array}$ & $16.9 \pm 4.8(15.8-17.9)$ & $15.4 \pm 5.7(14.2-16.6)$ & $14.8 \pm 5.5(13.1-16.4)$ & 2.853 & .050 & .027 \\
\hline $\begin{array}{l}\text { Salto longitudinal } \\
(\mathrm{cm})\end{array}$ & $102.8 \pm 21.2(98.7-107.1)$ & $104.5 \pm 21.5(99.7-109.1)$ & $96.1 \pm 17.7(89.7-102.2)$ & 2.371 & .096 & .022 \\
\hline $\begin{array}{l}\text { Course-Navette } \\
\text { (paliers) }\end{array}$ & $1.5(3.2-3.8)$ & $1.8(3.1-3.7)$ & $1.5(2.3-3.3)$ & 2.790 & .050 & .026 \\
\hline CF-ALPHA & $5.7 \pm 2.1(5.3-6.2)$ & $5.5 \pm 2.2(5.1-5.9)$ & $4.5 \pm 1.8(3.9-5.1)$ & 5.782 & .004 & .053 \\
\hline
\end{tabular}

Nota . PTD = Puntuación Total de Dificultades; CF-ALPHA = condición física.; $\mathrm{mCF}=$ menor condición física; $\mathrm{MCF}=$ mayor condición física.

\section{DISCUSIÓN}

Los resultados de este trabajo indican que la condición física está asociada de manera positiva con el bienestar emocional en escolares españoles de 7 a 12 años; y esto se demostró ya que aquellos con $\mathrm{MCF}\left(\mathrm{X} \geq \mathrm{P}_{50}\right)$ mostraron valores más positivos en las dimensiones de problemas de conducta $(p=.002)$, hiperactividad $(p<.001)$, problemas con compañeros $(p=.028)$ y conducta prosocial $(p<.001)$ y $\operatorname{PTD}(p=.029)$, en comparación con sus pares homólogos con $\mathrm{mCF}\left(\mathrm{X}<\mathrm{P}_{50}\right)$.

Además, los resultados muestran que esta relación podría ser bidireccional, ya que los escolares con niveles bajos de PTD $\left(\mathrm{X}<\mathrm{P}_{40}\right)$ presentaron una mejor condición física, es decir, un mejor desempeño físico en los test de 4 x $10 \mathrm{~m}$ $(p=.006)$, dinamometría manual $(p=.042)$, Course-Navette $(p=.050)$, y CF-ALPHA $(p=.008)$, en comparación con sus semejantes con nivel medio $\left(\mathrm{P}_{40} \leq \mathrm{X}>\mathrm{P}_{80}\right)$ o alto $\left(\mathrm{X} \geq \mathrm{P}_{80}\right)$ de PTD.

Con estos hallazgos se podría revelar la importancia de la condición física como elemento diferenciador en el bienestar emocional en la infancia y preadolescencia, lo que podría deberse a que poseer un mejor (o peor) estado de condición física puede modificar la forma en la que los jóvenes responden a sus emociones y se comportan consecuentemente (Berstein \& Mc Nally, 2017) -cabe anotar que los procesos subyacentes a esta relación continúan sin ser demostrados-.

Asimismo, estos hallazgos pueden ser de gran relevancia en la formación de las bases de la personalidad de los jóvenes, dado que identidades que no están formadas o conductas que no están consolidadas podrían verse influidas por influjos socioculturales, como los amigos, la familia, la publicidad o los medios de comunicación, entre otros, así como por estereotipos físicos vigentes (Ruiz-Pérez, Rioja-Collado, Graupera-Sanz, Palomo-Nieto \& García-Coll, 2015).

Por otro lado, se ha descrito que poseer un estado saludable de condición física constituye una respuesta integrada y eficaz de las estructuras y funciones que intervienen en la realización no solo de actividades físicodeportivas, sino también en el desarrollo de actividades físicas de la vida cotidiana (Gálvez et al., 2015b; Rosa et al., 2017), siendo la psiconeurológica una de las funciones más relevantes (Ortega et al., 2013). De igual forma, otras investigaciones han concluido que tener un mayor nivel de condición física se asocia con una mejor autopercepción en distintas variables relacionadas con el bienestar emocional negativo -como la ansiedad social-y positivo - como la felicidad subjetiva y la satisfacción vital- (Gálvez et al., 2016; Rodríguez-García et al., 2014; Rodríguez-García et al., 2015).

Adicionalmente, la condición física se también ha relacionado directamente con la competencia motriz, lo cual le permite al individuo con mejores cualidades físicas ser capaz de llevar a cabo tareas motrices complejas que, a su vez, lo hacen más eficaz y eficiente en el medio sociocultural en el que se desarrolla como persona, además de que le facilita incrementar la frecuencia y variedad de las actividades físicas, y amplía las oportunidades para tener éxito en sus relaciones sociales (Ruiz-Pérez et al., 2015).

A pesar de esto, la imposibilidad de encontrar investigaciones en la literatura científica que analicen la asociación entre la condición física y el bienestar emocional en escolares de primaria dificulta la discusión de los hallazgos de este estudio por medio de comparaciones directas; sin embargo, se puede afirmar que los resultados del presente trabajo coinciden parcialmente con los hallazgos reportados 
por Jeoung et al. (2013), en donde, con una muestra de 228 universitarios, se observó una fuerte asociación entre diversas variables determinantes del estado de condición física -como la fuerza de prensión manual, la resistencia aeróbica, la fuerza-resistencia muscular y la composición corporal- con distintas dimensiones que influyen en el bienestar emocional, especialmente en jóvenes y adultos -como la somatización, la psicosis, la paranoia, la fobia, la hostilidad, la ansiedad, la depresión, la sensibilidad interpersonal y la obsesión compulsiva $-(\beta$ no estandarizada entre -.535 y $.287 ; \mathrm{R}^{2}$ entre .048 y $.177 ; p$ entre $<.001$ y .032$)$.

En este mismo sentido, Padilla-Moledo et al. (2012) concluyeron, por medio de un estudio transversal con 684 participantes de 6 a 17.9 años (319 mujeres), que las personas jóvenes con mejor capacidad aeróbica (medida con el test de Course-Navette) presentaban una mayor probabilidad de poseer un bienestar emocional más positivo (evaluada a través del cuestionario Health Behavior in School-aged Children); y, de igual forma, Jiménez-Moral et al. (2013) observaron en 388 participantes de 12 a 18 años (207 mujeres) una relación significativa entre la capacidad aeróbica (medida con el test de Course-Navette) y algunos indicadores del bienestar emocional positivo, como la felicidad y la satisfacción vital (evaluadas mediante las escalas Subjective Happiness Scale y Satisfaction With Life Scale, respectivamente), siguiendo los puntos de corte establecidos por el FITNESSGRAM ${ }^{\circledR}$ (Cooper Institute for Aerobics Research, 1999).

Asimismo, otros estudios en línea con los resultados del presente trabajo muestran que: (a) el incremento en el nivel de condición física mediante la aplicación de intervenciones basadas en la realización de actividad física sistematizada (al menos 3 días/semana, 60 minutos/día, $\geq 3$ METS) se asocia fuertemente con una reducción de los síntomas en desórdenes psicosociales como la ansiedad y la depresión en adolescentes (Jeong et al., 2005), adultos (Hackney \& Earhart, 2010) y adultos mayores (Mavrovouniotis et al., 2010); (b) tras la aplicación de un programa basado en la práctica de yoga y danza africana en escolares que padecían estrés y emociones negativas hubo una reducción en los síntomas de dichas dificultades (West et al., 2004); (c) intervenciones basadas en la realización de ejercicio físico frecuentemente mejoraban la calidad de vida percibida en mujeres adolescentes con problemas psicológicos como el estrés y otras alteraciones psicosomáticas (Philipsson et al., 2013); (d) la actividad física escolar es una estrategia pedagógica que mejora el estado de salud física y el bienestar emocional (Gálvez et al., 2015a; Rosa et al., 2017a; 2017b); y (e) que propuestas que inciden en el análisis de las variables del estado de condición física pueden ser determinantes en diversos constructos relacionados con el bienestar emocional, como el autoconcepto (RodríguezGarcía et al., 2014), la salud autopercibida (Rosa-Guillamón \& García-Cantó, 2016), la calidad de vida (Gálvez et al., 2015a) y la satisfacción vital (Rosa et al., 2017a).

De estos resultados, derivados todos de investigaciones de corte transversal y longitudinal, podría concluirse que el uso lúdico y terapéutico de la actividad física favorecen no solo el desarrollo de los aspectos físicos, sociales, afectivos y creativos de las personas, sino también su equilibrio emocional; sin embargo, Biddle y Asare (2011) encontraron en un metaanálisis con muestras de niños y adolescentes que aunque la asociación entre actividad física y bienestar emocional es evidente, los efectos de la práctica física sobre los diversos indicadores estudiados -como la ansiedad, la depresión, el rendimiento cognitivo y la autoestima- son débiles o moderados, y que un factor asociado al bajo bienestar emocional tendría que ver más con los hábitos sedentarios.

Asimismo, en otro metaanálisis, Soyeon y Fedewa (2011) recopilaron trabajos que habían estudiado la relación entre diversos parámetros de la actividad física -como el tipo, intensidad, organización, frecuencia, tiempo de práctica, y responsable de la actividad - con diferentes dimensiones del bienestar emocional -como la autoestima, calidad de vida percibida, autoconcepto, ansiedad, depresión, problemas de conducta, problemas cognitivos, síntomas emocionales-, y encontraron distintos resultados en función de la metodología empleada y las características de los participantes, siendo los efectos de la actividad física sobre el bienestar emocional, en general, moderados.

Teniendo esto en cuenta, a pesar de los argumentos que apoyan la relación entre la condición física y el bienestar emocional, aún quedan diversos aspectos por ser aclarados en esta asociación, como es el caso de las explicaciones causales entre estas variables y el efecto de la mejora en los distintos parámetros de la condición física sobre las dimensiones del bienestar emocional. Además, podrían incluso realizarse investigaciones de control aleatorizado o longitudinales que fortalezcan los resultados encontrados, sobre todo, empleando métodos de medida más directos, como los acelerómetros, los cicloergómetros o los métodos enzimáticos colorimétricos, entre otros, además de la sensometría u otros métodos para evaluar las emociones.

Finalmente, cabe mencionar que este trabajo presenta limitaciones inherentes a los diseños de tipo transversal y al muestreo realizado, así como por la exclusión de otros parámetros potencialmente influyentes en la condición 
física, como el estatus socioeconómico - que puede influir en las condiciones de vida higiénica, como en los patrones de sueño y descanso, el nivel académico, las posibilidades de práctica deportiva, la alimentación, etc.- o el nivel de ejercicio físico - que podría influir en el nivel de condición física y la composición corporal, por ejemplo-.

En resumen, los resultados de este estudio sugieren una relación positiva y bidireccional entre la condición física y el bienestar emocional, por lo que se refuerza la necesidad de impulsar programas de mejora de la condición física para evaluar su efecto sobre las capacidades psicológicas de los escolares. En este sentido, deberían realizarse estudios longitudinales y prospectivos que determinen el modo en que la tendencia a poseer o no un mejor nivel de condición física puede intervenir sobre el bienestar emocional de los individuos a lo largo de su escolarización y en posteriores periodos vitales.

\section{REFERENCIAS}

Amstrong, L. E., Whaley, M. H., Brubaker, P. H., \& Otto, R. M. (2005). American College of Sport Medicine. Guidelines for Exercise Testing and Prescription (7a. ed.). Phyladelphia: Lippincott Williams \& Wilkins.

Babiss, L. A., \& Gangwisch, J. E. (2009). Sports participation as a protective factor against depression and suicidal ideation in adolescents as mediated by self-esteem and social support. Journal of Developmental and Behavioral Pediatrics, 30(5), 376-384. Doi: 10.1097/DBP.0b013e3181b33659.

Bernstein, E. E., \& McNally, R. J. (2017). Acute aerobic exercise helps overcome emotion regulation deficits. Journal of Cognition and Emotion, 31(4), 834-843. Doi: 10.1080/02699931.2016.1168284.

Biddle, S. J., \& Asare, M. (2011). Physical activity and mental health in children and adolescents: a review of reviews. British Journal Of Sports Medicine, 45(11), 886-895. Doi: http//:doi.org/10.1136/bjsports-2011-090185.

Blair, S. N. (2009). Physical inactivity: the biggest public health problem of the 21 st century. British Journal of Sports Medicine, 43(1), 1-2.

Cooper Institute for Aerobics Research (1999). FITNESSGRAM Test Administrator's Manual (2nd. ed.). Champaign, Il: Human Kinetics.

Dencker, M., Thorsson, O., Karlsson, M.K., Linden, C., Svensson, J., Wollmer, P., \& Andersen, L.B. (2006). Daily physical activity and its relation to aerobic fitness in children aged 8-11 years. European Journal of Applied Physiology, 96, 587-92. Doi: 10.1007/s00421-005-0117-1.

Diener, E., Suh, E. M., Lucas, R. E., \& Smith, H. L. (1999). Subjective well-being. Three decades of progress. Psycho- logical Bulletin, 125(2), 276-302. Doi: http://dx.doi. org/10.1037/0033-2909.125.2.276

Gálvez, A., Rodríguez-García, P. L., García-Canto, E., Rosa, A., Pérez Soto, J. J., Tárraga, M. L., \& Tárraga, P. J. (2015a). Capacidad aeróbica y calidad de vida en escolares de 8 a 12 años. Clínica e Investigación en Arteriosclerosis, 27(5), 239-245. Doi: https://doi.org/10.1016/j.arteri.2015.01.001

Gálvez, A., Rodríguez-García, P. L., Rosa, A., García-Cantó, E., Pérez-Soto, J. J., Tárraga, P. J., \& Tárraga, M. L. (2016). Capacidad aeróbica, estado de peso y autoconcepto en escolares de primaria. Clínica e Investigación en Arteriosclerosis, 28(1), 1-8. Doi: https://doi.org/10.1016/j.arteri.2015.10.002

Gálvez, A., Rodríguez-García, P. L., Rosa, A., García-Canto, E., Pérez Soto, J. J., Tárraga, M. L., \& Tárraga, P. J. (2015b). Nivel de condición física y su relación con el estatus de peso corporal en escolares. Nutrición Hospitalaria, 31(1), 393-400. Doi:10.3305/nh.2015.31.1.8074

Gálvez, A., Rosa, A., García-Cantó, E., Rodríguez-García, P. L., Pérez-Soto, J.J., Tárraga, M.L., \& Tárraga, P. J. (2015c). Estado nutricional y calidad de vida relacionada con la salud en escolares el sureste español. Nutrición Hospitalaria, 31(2), 737-743. Doi:10.3305/nh.2015.31.2.8468

Goodman, R. (1997). The Strengths and Difficulties Questionnaire: A Research Note. Journal of Child Psychology and Psychiatry, 38, 581-586. Doi: 10.1111/j.1469-7610.1997. tb01545.x.

Goodman, R., Ford, T., Simmons, H., Gatward, R., \& Meltzer, H. (2000a). Using the Strengths and Difficulties Questionnaire (SDQ) to screen for child psychiatric disorders in a community sample. British Journal of Psychiatry, 177, 5349. Doi: https://doi.org/10.1192/bjp.177.6.534

Goodman, R., Renfrew, D., \& Mullick, M. (2000b).Predicting type of psychiatric disorder from Strengths and Difficulties Questionnaire (SDQ) scores in child mental health clinics in London and Dhaka. European Child and Adolescent Psychiatry, 9, 129-34. Doi: 10.1007/s007870050008

Goodman, R., \& Scott. (1999). Comparing the Strengths and Difficulties Questionnaire and the Child Behavior Checklist: Is small beautiful? Journal of Abnormal Child Psychology, 27, 17-24. Doi: http//:doi.org/10.1023/A:1022658222914.

Hackney, M. E., \& Earhart, G. M. (2010). Social partnered dance for people with serious and persistent mental illness: a pilot study. The Journal of Nervous and Mental Disease, 198(1), 76-78. Doi: 10.1097/NMD.0b013e3181c81f7c.

Jeong, Y. J., Hong, S. C., Lee, M. S., Park, M. C., Kim, Y. K., \& Suh, C. M. (2005). Dance movement therapy improves emotional responses and modulates neurohormones in adolescents with mild depression. International Journal of Neuroscience, 115(12), 1711-1720. Doi: 10.1080/00207450590958574.

Jeoung, B., Hong, M, \& Lee, Y. (2013). The relationship between mental health and health-related physical fitness of 
university students. Journal of Exercise Rehabilitation, 9(6), 544-548. Doi: 10.12965/jer.130082.

Jiménez, M. G., Martínez, P., Miró, E., \& Sánchez, A. I. (2008). Bienestar psicológico y hábitos saludables: ¿están asociados a la práctica de ejercicio físico? International Journal of Clinical and Health Psychology, 8(1), 185-202.

Jiménez-Moral, J. A., Zagalaz, M. L., Molero, D., Pulido-Martos, M., \& Ruiz, J.R. (2013). Capacidad aeróbica, felicidad y satisfacción con la vida en adolescentes españoles. Revista de Psicología del Deporte, 22(2), 429-436.

Martínez-Vizcaíno, V., \& Sánchez-López, M. (2008). Relación entre actividad física y condición física en niños y adolescentes. Revista Española de Cardiología, 61(2), 108-11. Doi: 10.1157/13116196.

Mavrovouniotis, F. H., Argiriadou, E. A., \& Papaioannou, C.S. (2010). Greek traditional dances and quality of old people's life. Journal of Bodywork and movement therapies, 14(3), 209-218. Doi: 10.1016/j.jbmt.2008.11.005.

Navarro-Pardo, E., Meléndez, J. C., Sales, A., \& Sancerni, M. D. (2012). Desarrollo infantil y adolescente: trastornos mentales más frecuentes en función de la edad y el género. Psicothema, 24(3), 377-383.

Organización Mundial de la Salud (2013). World Happines Report. Recuperado de http://unsdsn.org/wp-content/ uploads/2014/02/ WorldHappinessReport2013 online.pdf.

Ortega, F. B., Artero, E. G., Ruiz, J. R., España-Romero, V., Jiménez-Pavón, D., Vicente-Rodríguez, G, ... \& Castillo, M. J. (2009). Physical fitness levels among European adolescents: The HELENA study. British Journal of Sports Medicine, 45, 20-29. Doi: 10.1136/bjsm.2009.062679.

Ortega, F. B., Ruiz, J., \& Castillo, M. J. (2013). Actividad física, condición física y sobrepeso en escolares y adolescentes: evidencia procedente de estudios epidemiológicos. Endocrinología y Nutrición, 60(8), 458-469. Doi: 10.1016/j. endonu.2012.10.006.

Ortega, F. B., Ruiz, J. R., Castillo, M. J., Moreno, L. A., González-Gross, M., Warnberg, J, ... \& Gutiérrez, A. (2005). Low level of physical fitness in Spanish adolescents. Relevance for future cardiovascular health (AVENA study). Revista Española de Cardiología, 58, 898-909.

Padilla-Moledo, C., Castro-Piñero, J., Ortega, F. B., Mora, J., Márquez, S., Sjöström, M., \& Ruiz, J. R. (2012). Positive health, cardiorespiratory fitness and fatness in children and adolescents. The European Journal of Public Health, 22, 52-56. Doi: http//:doi.org/10.1093/eurpub/ckr005.

Panagiotopoulos, C., Ronsley, R., Al-Dubayee, M., Brant, R., Kuzeljevic, B., Rurak, E., \& Masse, L. C. (2011). The Centre for Healthy Weights-Shapedown BC: A Family-Centered, Multidisciplinary Program that Reduces Weight Gain in Obese Children over the Short-Term. International Journal of Environmental Research and Public Health, 8(12), 4662-4678. Doi: 10.3390/ijerph8124662.
Philipsson, A., Duberg, A., Moller, M., \& Hagberg, L. (2013). Cost-utility analysis of a dance intervention for adolescent girls with internalizing problems. Cost Effectiveness and Resource Allocation, 11(1), 4. Doi: http//:doi. org/10.1186/1478-7547-11-4.

Rodríguez-García, P. L., Gálvez, A., García-Cantó, E., PérezSoto, J. J., Rosa, A., Tárraga, L., \& Tárraga, P. L. (2015) Relationship between the Self-Concept and Muscular Strength in Southern Spanish Children. Journal of Psychology \& Psychotherapy, 5, 222. Doi: http://dx.doi.org/10.4172/21610487.1000222

Rodríguez-García, P. L., Tárraga, L., Rosa, A., García-Cantó, E., Pérez-Soto, J. J., Gálvez, A., \& Tárraga, P. (2014). Physical Fitness Level and Its Relationship with Self-Concept in School Children. Psychology, 5, 2009-2017. Doi: 10.4236/ psych.2014.518204.

Rosa-Guillamón, A., \& García-Cantó, E. (2016). Relación entre condición física y salud mental en escolares de primaria. Revista iberoamerican de Ciencias de la actividad fisica y el deporte, 5(2), 31-42.

Rosa, A., Rodríguez-García, P. L., García-Cantó, E., \& PérezSoto, J. J. (2015). Niveles de condición física de escolares de 8 a 11 años en relación al género y a su estatus corporal. Ágora para la EF y el Deporte, 17(3), 237-250.

Rosa, A., García-Cantó, E., Rodríguez-García, P. L., \& Pérez-Soto, J. J. (2016). Physical condition and quality of life in schoolchildren aged between 8 and 12. Revista de la Facultad de Medicina, 65(1), 37-42. Doi: http://dx.doi. org/10.15446/revfacmed.v65n1.59634.

Rosa, A., García-Cantó, E., Rodríguez-García, P. L., \& PérezSoto, J. J. (2017a). Weight status, physical fitness and satisfaction with life among elementary school children. A pilot study. MH Salud Revista en Ciencias del Movimiento Humano y Salud, 13(2), 1-15. Doi: http//:doi.org/10.15359/ mhs.13-2.2.

Rosa, A., García-Cantó, E., Rodríguez-García, P. L., Pérez-Soto, J. J., Tárraga, M. L., \& Tárraga, P. J. (2017b). Physical activity, physical fitness and quality of diet in schoolchildren from 8 to 12 years. Nutrición Hospitalaria, 34, 12921298. Doi: 10.20960/nh.813.

Ruchkin, V., Jones, S., Vermeiren, R., \& Schwab-Stone, M. (2008). The Strengths and Difficulties Questionnaire: The Self-Report Version in American Urban and Suburban Youth. Psychological Assessment, 20, 175-182. Doi: 10.1037/1040-3590.20.2.175.

Ruiz, J. R., España Romero, V., Castro Piñero, J., Artero, E. G., Ortega, F. B., Cuenca, M, ... \& Castillo, M. J. (2011). Batería ALPHA-Fitness: test de campo para la evaluación de la condición física relacionada con la salud en niños y adolescentes. Nutrición Hospitalaria, 26(6), 1210-1214. Doi:10.3305/nh.2011.26.6.5611.

Ruiz-Pérez, L. M., Rioja-Collado, N., Graupera-Sanz, J. L., Palomo-Nieto, M., \& García-Coll, V. (2015). GRAMI-2: desarrollo de un test para evaluar la coordinación motriz 
global en la educación primaria. Revista Iberoamericana de Psicología del Ejercicio y el Deporte, 10(1), 103-111. Doi: $\mathrm{http} / /$ :doi.org/10.5232/ricyde2017.04907.

Soyeon A., \& Fedewa, A.L. (2011). A Meta-analysis of the Relationship Between Children's Physical Activity and Mental Health. Journal of Pediatric Psychology, 1(11), 1-13. Doi:10.1093/jpepsy/jsq107.
Thomas, J. R., \& Nelson, J. K. (2007). Métodos de investigación en actividad física. Barcelona: Paidotribo.

West, J., Otte, C., Geher, K., Johnson, J., \& Mohr, D. (2004). Effects of hatha yoga and african dance on perceived stress, affect, and salivary cortisol. Annals of Behavioral Medicine, 28(2), 114-118. Doi: 10.1207/s15324796abm2802_6 